\title{
Etude sérologique et bactériologique des mycoplasmoses aviaires dans la région du Cap Bon en Tunisie
}

\author{
M. Boussetta ${ }^{1}$ N. Chaouachi ${ }^{2}$ B. Mlik ${ }^{1}$
}

\begin{abstract}
Mots-clés
Poulet de chair - Poule pondeuse Dindon - Animal reproducteur . Mycoplasmose - Mycoplasma gallisepticum - Mycoplasma synoviae M ycoplasma meleagridis - Immunologie - Bactériologie - Tunisie.
\end{abstract}

\begin{abstract}
Résumé
Une enquête séroépidémiologique et bactériologique a été effectuée en Tunisie dans une région à forte concentration aviaire pour le dépistage des mycoplasmoses à Mycoplasma gallisepticum, M. synoviae chez la poule et chez la dinde. Au cours de l'enquête, 63 élevages ont été visités et 780 prélèvements de sang et écouvillons trachéaux ont été effectués et analysés pour la recherche sérologique d'anticorps anti-Mycoplasma gallisepticum, $-M$. synoviae et $-M$. meleagridis. La recherche bactériologique a porté sur $M$. gallisepticum et $M$. synoviae. Les taux d'infection des troupeaux déterminés par sérologie étaient de 36,5 p. 100 pour $M$. gallisepticum, de 19 p. 100 pour $M$. synoviae et de 0 p. 100 pour M. meleagridis. Les élevages positifs en bactériologie pour $M$. gallisepticum et $M$. synoviae étaient respectivement au nombre de 15 et 5 , soit 23,8 et 7,9 p. 100 des élevages visités.
\end{abstract}

Les mycoplasmoses aviaires à Mycoplasma gallisepticum et Mycoplasma synoviae sont responsables de troubles respiratoires et articulaires chez la poule, la dinde et chez d'autres espèces aviaires $(26,27)$. Ces infections engendrent des conséquences économiques importantes dans tous les secteurs de production en aviculture : retard de croissance pour la filière chair et baisse de production d'œufs pour les pondeuses et reproductrices (14). L'antibiothérapie est coûteuse et les molécules actives sur les mycoplasmes sont peu nombreuses (8). A la connaissance des auteurs, aucune étude sur la mycoplasmose clinique en Tunisie n'a été publiée. Les animaux prélevés au cours de cette enquête étaient d'apparence saine. Cette étude a concerné tous les secteurs de production avicole rencontrés au Cap Bon, région à forte concentration aviaire. Elle a porté sur la recherche des anticorps anti-Mycoplasma par la technique de l'agglutination rapide sur lame (18) et ELISA (2), ainsi que sur l'isolement de Mycoplasma gallisepticum et Mycoplasma synoviae sur des milieux de culture spécifiques $(5,10)$.

1. Laboratoire des mycoplasmes, Institut Pasteur de Tunis, 13 Place Pasteur, BP 74, 1002 Tunis-Belvédère, Tunisie

Tél: (216-1) 783022 ; Fax : (216-1) 791833

2. Docteur vétérinaire, 5 rue Ali Annabi, 1002 Tunis-Belvédère, Tunisie
MATERIEL ET METHODES

\section{Enquête sur le terrain}

\section{Région}

L'étude a concerné six délégations (ou zones administratives) de la région du Cap Bon où la concentration avicole industrielle était importante (figure 1). Le cheptel aviaire dans cette région était estimé à 7 millions de volailles, réparti comme suit (11) : 80 p. 100 de poulets de chair, 9 p. 100 de poules pondeuses, 9 p. 100 de dindes, 1 p. 100 de poules reproductrices et 1 p. 100 de poulets traditionnels.

\section{Questionnaire}

La visite de chaque élevage a été reportée sur une fiche de renseignements. Les informations ont été recueillies auprès des éleveurs et concernaient les antécédents pathologiques, le type de production, la prophylaxie, l'antibiosupplémentation et l'antibiothérapie.

Les élevages visités étaient au nombre de 63, répartis sur les six délégations de la région, à raison d'une dizaine d'élevages par délégation. Le choix a été effectué de façon à couvrir tous les types de production (chair, ponte, reproducteurs) ainsi que l'élevage traditionnel. 


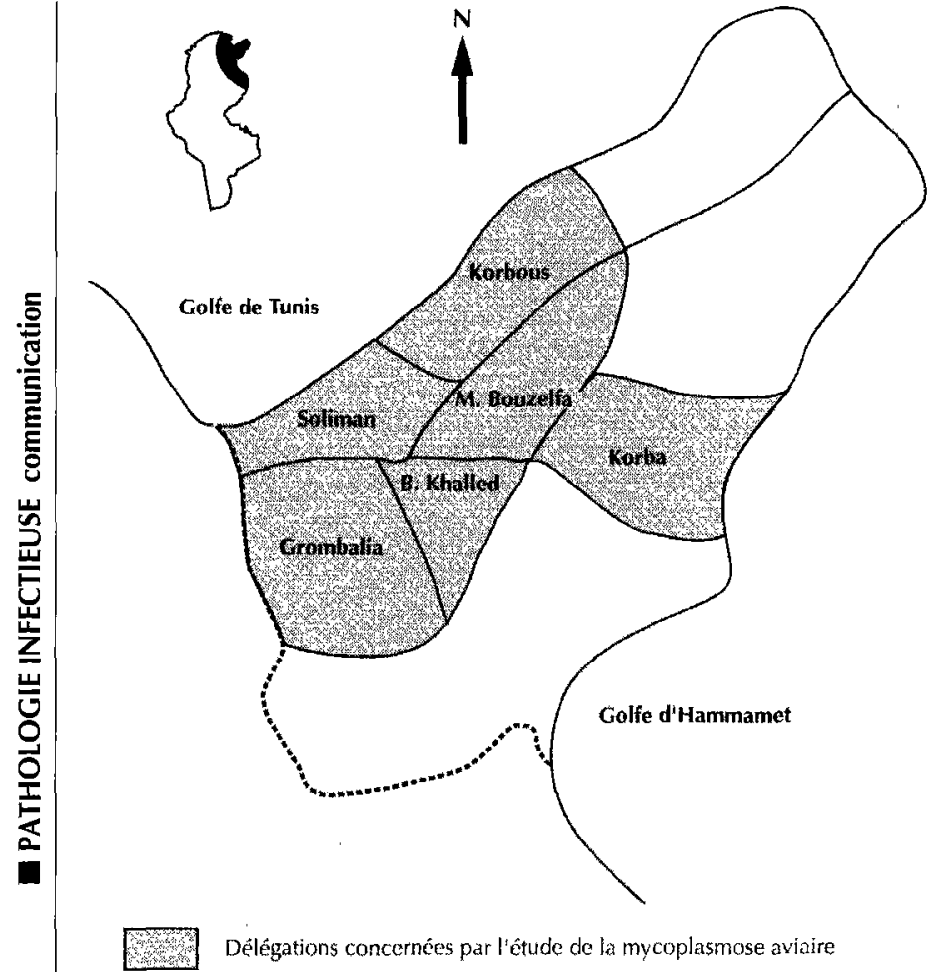

Figure 1 : carte géographique de la région du Cap Bon, Tunisie.

\section{Prélèvements}

Pour chaque sujet, un écouvillon trachéal destiné à l'analyse bactériologique (25) et une prise de sang pour la recherche des anticorps anti-Mycoplasma $(1,16,21)$ ont été réalisés simultanément. Le transport et la conservation des échantillons ont été effectués à $+4^{\circ} \mathrm{C}$.

\section{Examens de laboratoire}

\section{Sérologie}

L'analyse sérologique a consisté, dans un premier temps, à rechercher les anticorps dirigés contre les trois cspc̀ces de mycoplasmes : Mycoplasma gallisepticum, $M$. synoviae et $M$. meleagridis, selon la technique d'agglutination rapide sur lame $(\operatorname{ARL})(6,20)$ en utilisant les antigènes spécifiques (Intervet, France). Dans un deuxième temps, la détection des anticorps s'est orientée vers la souche de mycoplasme prédominante en ARL selon la méthode immunoenzymatique ELISA Mycoplasma gallisepticum, KPL (Proflock, USA) (15).

\section{Bactériologie}

Le milieu d'isolement utilisé était le FM4 décrit par Frey (7). Ce milieu a été comparé à celui des laboratoires Unipath (Oxoid) : CM 403 pour le milieu liquide et CM 401 pour le milieu solide avec le supplément sélectif SR 0596. Tous ces milieux ont été complétés avec de la L-cystéine $(0,05$ p. 100), de la nicotine amide dinucléotide (Sigma), et les vitamines BME 100X (Sigma).

Les souches de référence utilisées étaient: Mycoplasma gallisepticum ATCC 15302, Mycoplasma synoviae WVU 1853 et Mycoplasma meleagridis ATCC 25294. Les sérums monospécifiques de référence correspondants étaient produits chez le lapin et provenaient du Laboratoire national de pathologie aviaire (Ploufragan, France).
L'identification des cultures positives a été effectuée après caractérisation biochimique $(3,4,19)$ et étude des anticorps par immunofluorescence indirecte $(9,22)$ en utilisant un sérum anti-lapin marqué à la fluorescéine (Sigma).

Les caractères étudiés étaient : le métabolisme du glucose ou de l'arginine, la réduction des sels de tétrazolium, l'activité de la phosphatase alcaline, la formation de films et spots et le test de croissance en présence de digitonine.

\section{RESULTATS}

\section{Sérologie}

Le dépistage sérologique des troupeaux par agglutination sur lame (tableau I) a montré la présence d'anticorps antiMycoplasma gallisepticum et $-M$. synoviae avec des taux respectifs de 36,5 et 19 p. 100 . La sérologie est restée négative pour Mycoplasma meleagridis. L'analyse des sérums pour la recherche des anticorps anti-Mycoplasma gallisepticum a montré une corrélation de 97,7 p. 100 entre les techniques ARL et ELISA. En effet, sur les 176 sérums positifs pour Mycoplasma gallisepticum, 172 étaient positifs par la technique ELISA. Aucun des autres sérums ne s'est révélé positif en ELISA. La totalité des poulets traditionnels présentaient des anticorps anti-Mycoplasma. Quelques sérums chez la dinde et la poule reproductrice se sont révélés positifs pour Mycoplasma gallisepticum. Tous les secteurs de production (y compris celui de la dinde) étaient contaminés par M. gallisepticum (tableau II).

Le tableau III montre la répartition des prélèvements positifs de $M$. gallisepticum et $M$. synoviae selon les délégations. Les taux d'infections étaient variables en fonction des zones.

\section{Bactériologie}

Les milieux de culture FM4, Oxoid, solide et liquide ont donné des résultats similaires. Les cultures positives étaient au nombre de 43 pour Mycoplasma gallisepticum et 11 pour Mycoplasma synoviae sur les 63 élevages visités, soit respectivement un taux de 23,8 et 7,9 p. 100 (tableaux II et III). Mycoplasma gallisepticum n'a pas été isolée chez la dinde, ni chez les reproducteurs. Cependant la totalité des élevages de poulets fermiers, $11 \mathrm{de}$ poules pondeuses et 5 de poulets de chair ont montré des cultures positives. Toutes les délégations étaient contaminées par $M$. gallisepticum, mais deux (Korba et Korbous) étaient indemnes de $M$. synoviae (tableau III).

\section{Tableau I}

Résultats sérologiques et bactériologiques des mycoplasmoses aviaires chez la poule et la dinde

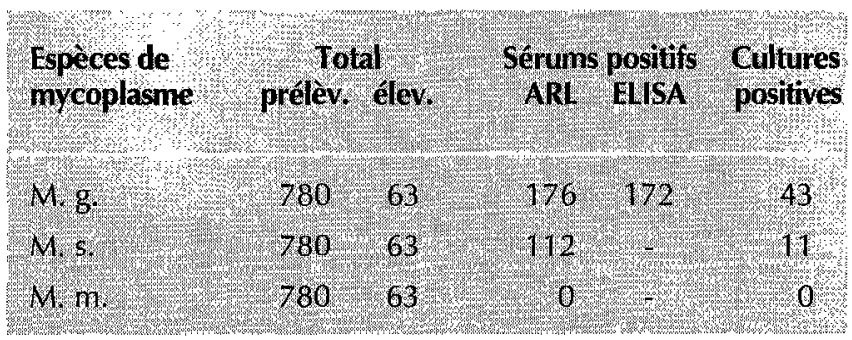

M. g. : Mycoplasma gallisepticum; M. s. : Mycoplasma synoviae;

M. m. : Mycoplasma meleagridis ; - ; non réalisé 
Tableau II

Répartition des échantillons positifs en fonction des secteurs de production

\begin{tabular}{|c|c|c|c|c|c|c|}
\hline \multirow[t]{2}{*}{ Types de production } & \multirow[t]{2}{*}{$\begin{array}{c}\text { N bre d'élevages } \\
\text { visités }\end{array}$} & \multirow[t]{2}{*}{$\begin{array}{c}\text { Nbre de } \\
\text { prélèvements }\end{array}$} & \multicolumn{2}{|c|}{$\begin{array}{l}\text { Elev. positifs } \\
\text { en sérolologie }\end{array}$} & \multicolumn{2}{|c|}{$\begin{array}{l}\text { Elev. positifs } \\
\text { en culture }\end{array}$} \\
\hline & & & M. g. & M. s. & M. g. & M. s. \\
\hline Poulets traditionnels & 5 & 25 & 5 & 5 & 5 & 2 \\
\hline Poulets de chair & 9 & 93 & 5 & 1 & 5 & 1 \\
\hline Poules pondeuses & 21 & 181 & 11 & 6 & 5 & 2 \\
\hline Poules reproductrices & 12 & 174 & 1 & 0 & 0 & 0 \\
\hline Dindes & 16 & 307 & 1 & 0 & 0 & 0 \\
\hline Total & 63 & 780 & 23 & 12 & 15 & 5 \\
\hline$\%$ élevages positifs & & & $36,5 \%$ & $19 \%$ & $23,8 \%$ & $7,9 \%$ \\
\hline
\end{tabular}

M. g. : Mycoplasma gallisepticum; M. s. : Mycoplasma synoviae

\section{Tableau III}

Répartition des échantillons positifs par délégation et nombre d'élevages contaminés

\begin{tabular}{lccccc} 
Délégations & $\begin{array}{c}\text { N bre d'élev. } \\
\text { visités }\end{array}$ & $\begin{array}{c}\text { N bre d'élev. } \\
\text { positifs } \\
\text { en sérololgie } \\
\text { M. g. M. s. }\end{array}$ & $\begin{array}{c}\text { N bre d'élev. } \\
\text { positifs } \\
\text { en culture } \\
\text { M. g. }\end{array}$ \\
\hline Korba & 8 & 1 & 0 & 1 & 0 \\
Korbous & 10 & 1 & 0 & 1 & 0 \\
Soliman & 10 & 6 & 5 & 3 & 2 \\
M. Bouzelfa & 11 & 5 & 3 & 1 & 1 \\
B. Khalled & 10 & 5 & 2 & 4 & 1 \\
Grombalia & 14 & 5 & 2 & 5 & 1 \\
\hline Total & 63 & 23 & 12 & 15 & 5 \\
\hline $\begin{array}{l}\text { \% d'élevages } \\
\text { positifs }\end{array}$ & & $36,5 \%$ & $19 \%$ & $23,8 \%$ & $7,9 \%$ \\
\end{tabular}

M. g. : Mycoplasma gallisepticum; M. s. : Mycoplasma synoviae

\section{DISCUSSION}

Cette étude a permis d'évaluer les taux d'infection mycoplasmique dans la région du Cap Bon. Tous les types de production aviaire et toutes les zones de l'étude ont présenté des anticorps antiMycoplasma gallisepticum. La bactériologie a montré la présence de Mycoplasma gallisepticum et de Mycoplasma synoviae dans les élevages traditionnels, dans les élevages de poulets de chair et ceux de pondeuses. La poule reproductrice et la dinde paraissaient mieux protégées. L'élevage traditionnel et celui du poulet de chair étaient fortement contaminés et pouvaient ainsi constituer un risque de contamination perpétuel de l'environnement, surtout pour M. gallisepticum. Les régions de Korba et Korbous étaient indemnes de M. synoviae. Ces deux zones sont en effet en bordure de mer et ont donc moins de zones limitrophes que les autres délégations de cette enquête. La discordance entre sérologie positive et culture négative peut s'expliquer par l'intervention de plusieurs facteurs : l'utilisation massive d'une antibio-supplémentation pour pallier aux différentes insuffisances de conduite des élevages et aux interventions stressantes (vaccination, débecquage, transfert de bâtiments), aux écouvillons pauvres en germes, germes fragiles et peu viables (27), et à la présence d'inhibiteurs tissulaires de croissance des mycoplasmes (13).

La recherche de mycoplasmes pourrait s'étendre aux poussins d'un jour et démarrer à partir de l'œuf embryonné $(17,23)$.

Cette étude mérite d'être élargie à d'autres régions du pays pour établir une carte permettant de définir et d'identifier les mycoplasmes circulants dans les élevages tunisiens et, par la même occasion, pour servir de base à la surveillance épidémiologique.

\section{CONCLUSION}

Dans les 63 élevages concernés par cette étude, la présence de Mycoplasma gallisepticum et de Mycoplasma synoviae dans les différentes productions avicoles en Tunisie a été montrée par l'analyse sérologique et bactériologique. Le taux d'infection moyen des troupeaux visités était de 36,5 p. 100 pour Mycoplasma gallisepticum et de 19 p. 100 pour Mycoplasma synoviae en sérologie, alors qu'il était respectivement de 23,8 et 7,9 p. 100 pour l'isolement. Cette étude n'a pas révélé la présence de Mycoplasma meleagridis.

\section{Remerciements}

Les auteurs remercient les Drs vétérinaires I. Choura, A. Jelailia, B. Rabaa, A. Abbas pour leur collaboration sur le terrain. Ils remercient également le Secrétariat d'Etat tunisien à la Recherche scientifique et technique pour l'aide financière.

\section{BIBLIO GRAPH IE}

1. AVAKIAN A.P., KLEVEN S.H., 1990. The humoral immune response of chickens to Mycoplasma gallisepticum and Mycoplasma synoviae studied by immunoblotting. Vet. Microbiol., 24: 155-169.

2. AVAKIAN A.P., KLEVEN S.H., GLISSON R., 1988. Evaluation of the specificity and sensitivity of two commercial enzyme linked immunosorbent assay kits, the serum plate agglutination test, and the hemagglutination inhibition test for antibodies formed in response to Mycoplasma gallisepticum. Avian Dis., 32: 262-272. 
3. BAAS E.J., JASPER D.E., 1972. Agar block technique for identification of Mycoplasma by use of fluorescent antibody. Appl. Microbiol., 23: 1097-1100.

4. BRADBURY J.M., 1977. Rapid biochemical tests for characterization of the mycoplasmatales. J. clin. Microbiol., 5: 531-534.

5. CHRISTENSEN N.H., YAVARI C.A., MCBAIN A.J., BRADBURY J.M., 1994. Investigation into the survival of Mycoplasma gallisepticum Mycoplasma synoviae and Mycoplasma iowae on materials found in the poultry house environment. Avian pathol., 23: 127-143.

6. DUPY N., SILIM A., BRU GERE-PICOUX J., 1994. Influence de différents traitements du jaune d'œufs sur le titrage des anticorps vitellins dirigés contre quatre virus aviaires par la technique Elisa. Rec. Méd. vét., $170: 847-855$.

7. FREY M.C., HAUSON R.P., ANDERSON D.P., 1968. A medium for the isolation of avian Mycoplasma. Am. J. vet. Res., 29: 2164-2171.

8. GAILLARD-PERRIN G., 1984. Les infections mycoplasmiques aviaires. Rec. Méd. vét., $160: 969-982$.

9. GARDELLA R.S., DELGINDRICE R.A., TULLY J.G., 1983. Immunofluorescence. Methods mycoplasmol., 1: 431-439.

10. GIBBS P.S., KLEVEN S.H., JACKWOOD M.W., 1994. Analysis and caracterisation of Mycoplasma gallisepticum isolates from Pennsylvania. Avian Dis., 38: 475-482.

11. GROUPEMENT INTERPROFESSIONNEL DES PRODUITS AVICO LES, 1995. Rapport annuel. Tunis, Tunisie, Groupement interprofessionnel des Produits avicoles.

12. JORDAN F.T.W., 1983. Avian mycoplasmas. In: Tully J.G. Whitcomb R.F. eds., The mycoplasmas, vol. 2. New york, USA Academic Press, p. 1-48.

13. KEMPF I., 1992. Mycoplasmoses aviaires. In : Brugère-Picoux J., Silim A. eds, Manuel de pathologie aviaire. Maisons-Alfort, France, ENVA, p. 205-217.

14. KEMPF I., CACON P.M., GUITTET M., OLLIVIER C., MORIN M. L'HOSPITALIER R., BENNEJEAN G., 1988. Mycoplasmose à Mycoplasma gallisepticum : réalisation d'un modèle expérimental, rôle de l'ammoniac comme facteur d'exacerbation. Avian pathol., 17: 601-616.

15. KEM PF I., GESBERT F., GUITTET M., BEN N EJEAN G., STIPKO VITS L., 1994. Evaluation of two commercial enzyme-linked immunosorbent assay kits for the detection of Mycoplasma gallisepticum antibodies. Avian pathol., 23: 329-338.

\section{Summary}

Boussetta M., Chaouachi N., Mlik B. Serological and bacteriological study of avian mycoplasmosis in the Cap Bon region of Tunisia

A Mycoplasma gallisepticum and $M$. synoviae seroepidemiological and bacteriological survey was performed on hens and turkeys in a region of Tunisia with heavy poultry concentration. During the survey 63 farms were visited and 780 blood samples and tracheal swabs were collected, and antibodies directed against $M$. gallisepticum, M. synoviae and $M$. meleagridis were searched. The bacteriological investigation concerned $M$. gallisepticum and $M$. synoviae. Serology of the flocks revealed $36.5,19$ and $0 \%$ infection rates with $M$. gallisepticum, M. synoviae and M. meleagridis, respectively. There were 15 flocks bacteriologically positive for M. gallisepticum and 5 for M. synoviae, i.e. 23.8 and $7.9 \%$ of the farms surveyed, respectively.

Key words: Broiler chicken - Layer chicken - Turkey Breeding stock - Mycoplasmosis - Mycoplasma gallisepticum Mycoplasma synoviae - Mycoplasma meleagridis Immunology - Bacteriology - Tunisia.
16. KLEVEN S.H., 1975. Antibody response to avian mycoplasmas. J. vet. Res., 36: 563-564

17. MOHAMMED H.O., YAMAMOTO R., CARPENTIER T.E., ORTMAYER H.B., 1986. Comparison of egg yolk and serum for the determination of Mycoplasma gallisepticum and Mycoplasma synoviae antibodies by enzyme-linked immunosobent assay. Avian Dis., 30: 398-408.

18. NOUGAYREDE P., TOQUIN D., ANDRAL B., GUITTET M., 1984 Dépistage sérologique des mycoplasmoses aviaires : agglutination rapide sur lame, inhibition de I'hémagglutination, inhibition métabolique, techniques appliquées au diagnostic des infections à Mycoplasma gallisepticum. Avian pathol., 13 : 753-768.

19. OFFICE INTERNATIONAL DES EPIZO OTIES, 1992. Manual of standards for diagnostic tests and vaccines, 2nd ed. Paris, France, OIE, p. 393-399.

20. PERRIN G., BENNEJEAN G., 1982. Le dépistage de l'infection mycoplasmique aviaire. Bull. Lab. Serv. vét., 5 : 13-29.

21. ROBERTS D.H., 1969. Serological response produced in chickens by three strains of Mycoplasma gallisepticum. J. Appl. Bacteriol., 32: 395-401.

22. ROSENDAL S., BLACK F.T., 1972. Direct and indirect immunofluorescence of unfixed and fixed Mycoplasma colonies. Acta pathol. microbiol. scand., section B, 80: 615-622.

23. TALKINGTON F.D., KLEVEN S.H., BROWN J., 1985. An enzymelinked immunosorbent assay for the detection of antibodies to Mycoplasma gallisepticum in experimentally infected chickens. Avian Dis., 29: 53-70

24. VARDAMAN T.H., YODER H.W.JR., 1971. Determination of non specific serological reactions to avian Mycoplasma antigens. Poult. Sci., 50: 183-186.

25. YODER H.W. Jr., 1991. Mycoplasma gallisepticum infection. In: Calnek B.W., Barnes H.J., Beard C.W., Reid W.M. Yoder H.W. Jr. eds., Diseases of poultry, 9th ed. Ames, lowa, USA, lowa State University Press, p. 198-212.

26. YOGEV D., LEVISOHN S., RAZIN S., 1989. Genetic and antigenic relatedness between Mycoplasma gallisepticum and Mycoplasma synoviae. Vet. Microbiol., 19: 57-84.

27. ZAIN Z.M., BRADBURY J.M., 1995. The influence of type of swab and laboratory method on the recovery of Mycoplasma gallisepticum and Mycoplasma synoviae in broth medium. Avian pathol., 24: 707-716.

Reçu le 5.3.97, accepté le 30.9.97

\section{Resumen}

Boussetta M., Chaouachi N., Mlik B. Estudio serológico y bacteriológico de las micoplasmosis avícolas en la región del Cabo, en Túnez

Se llevó a cabo una encuesta sero-epidemiológica y bacteriológica en Túnez, en una región de fuerte concentración avícola, para la detección de micoplasmosis por Mycoplasma gallisepticum y $M$. synoviae en la gallina y el pavo. Durante el estudio, se visitaron 63 establecimientos y se tomaron 780 muestras de sangre y frotis traqueales, los cuales se analizaron para la búsqueda serológica de anticuerpos anti $\mathrm{M}$. gallisepticum, M. synoviae y $M$. meleagridis. La búsqueda bacteriológica se realizó en $M$. gallisepticum y $M$. synoviae. Las tasas de infección de los hatos determinados mediante serología fueron de 36,5\% para $M$. gallisepticum, de $19 \%$ para $M$. synoviae y de $0 \%$ para $M$. meleagridis. Les establecimientos bacteriológicamente positivos para $M$. gallisepticum y $M$. synoviae fueron respectivamente 15 y de 5 , es decir de $23,8 \%$ y de $7,9 \%$ de los establecimientos visitados.

Palabras clave: Pollo de engorde - Gallina ponedora - Pavo Animal reproductor - Micoplasmosis - Mycoplasma gallisepticum - Mycoplasma synoviae - Mycoplasma meleagridis - Immunología - Bacteriología - Túnez. 